\title{
Comment appliquer la psychologie évolutionniste à l'étude des textes littéraires?
}

\author{
Marc Lapprand \\ Université de Victoria
}

Vous savez ce que c'est que le mot et que l'idée d'Évolution, que, depuis une vingtaine d'années, ils ont envahi, l'une après l'autre, pour les transformer ou les renouveler, toutes les provinces de l'érudition et de la science puisque nous savons ce que l'histoire naturelle générale, ce que l'histoire, ce que la philosophie en ont déjà tiré de profit, je voudrais examiner si l'histoire littéraire et la critique ne pourraient pas aussi l'utiliser à leur tour.

Ferdinand Brunetière, 1898, cité par Appleman, p. 571.

Il n'a pas fallu beaucoup de temps après L'Origine des espèces (1859) pour que le monde littéraire se penche sur la question 
d'une utilisation éventuelle des grands principes darwiniens d'évolution, d'adaptation et de sélection au profit de la critique littéraire. Dès The Descent of Man (1871), Darwin entrevoit que la propension aux arts est liée à la sélection sexuelle, procurant ainsi à ses porteurs une meilleure adaptation ${ }^{1}$. Si des idées originales ont vu le jour avant le tournant du dix-neuvième siècle, on ne peut pas affirmer pour autant qu'elles posaient les bases d'une véritable épistémologie darwinienne autour de l'objet littéraire. Ainsi par exemple de John Addington Symonds, qui en 1890 proposait de manière ultra-concise une application des principes de l'évolution à l'art et la littérature. En fait, il partait simplement de l'analogie entre une espèce vivante et un genre artistique d'une époque donnée, pour montrer que, conformément aux lois biologiques gouvernant toute espèce vivante, un genre littéraire va évoluer pour tendre à la perfection, puis décliner et, enfin, probablement disparaître. Il prend comme exemple le théâtre élisabéthain, démontre que son "germe» se trouve dans les Miracles du moyen-âge, comment il triomphe sous la plume de Marlowe puis de Shakespeare, pour enfin subir une dégradation progressive :

For him [the Evolutionist], this type of art exhibits qualities analogous to those of an organic complex undergoing successive phases of germination, expansion, efflorescence, and decay, which were independent of the volition of the men who effected them. (p. 611)

On le voit, au-delà de la panoplie terminologique obligée, affleure tout de même la notion de mutation aléatoire. Nonobstant, on est encore loin d'une théorie, et ce que propose

\footnotetext{
1 «In Europe the men of the Bronze period were supplanted by a more powerful and, judging from their sword-handles, larger-handed race; but their success was probably due in a much higher degree to their superiority in the arts. » (Ridley, p. 150)
} 
Symonds ressortit moins à une nouvelle méthodologie qu'à un simple ajustement à la mode darwinienne. Dans le même esprit, il eût pu se contenter de la forme poétique qu'est le sonnet, qui, $\mathrm{au}$ contraire du théâtre élisabéthain, est toujours en vie, notamment dans les langues latines, après quelque six siècles d'existence, son germe en ayant été posé par l'Italien Pétrarque, et son efflorescence ayant triomphé dans le Canzoniere (1470).

Comment le biologiste et naturaliste que fut Darwin a-t-il, à son corps défendant, pu devenir le promoteur d'une science appliquée au champ littéraire, voilà quelle pourrait être notre première question². Selon Michael Ghiselin (p. 43), la force de son approche est de constamment relier des éléments apparemment disparates, d'appliquer la méthode hypothéticodéductive à tout ce qu'il observe et de toujours combiner à la découverte la rigueur de la vérification ${ }^{3}$. Darwin est donc un créateur de système, ce que Foucault appelait naguère un "fondateur de discursivité », au même titre que Marx et Freud, par exemple. «La bévue généralement commise tourne autour $\mathrm{du}$ fait d'avancer que l'adaptation (fitness) d'un organisme signifie, dans son sens le plus pratique, non pas le fait de survivre, mais sa probabilité.» (Ghiselin, p. 64) La nature n'a pas de plan préconçu; elle s'organise par sauts dans le futur, selon le principe d'occasions réussies ou ratées, qui favorisent éventuellement l'adaptation de l'espèce.

2 D'autant plus que Darwin semble porter à la littérature un intérêt relativement modéré : " novels which are works of the imagination, though not of a very high order, have been for years a wonderful relief and pleasure to me, and I often bless all novelists » (Barlow, p. 113).

3 Il est par ailleurs confondant de voir avec quelle humilité Darwin parle luimême de ses erreurs et rectifications passées: il oppose aux critiques violentes qu'on a pu lui faire un travail acharné, avec pour seul souci l'intégrité scientifique (voir Barlow, p. 103-104). 
Depuis Aristote subsiste dans la doxa occidentale une fâcheuse confusion entre fonction et utilité. Ghiselin illustre cette confusion par l'exemple de la roulette russe : le revolver est parfaitement adapté à cette fonction, mais il n'a pas été conçu pour cet usage particulier. La fonction n'implique donc logiquement pas une raison d'être préalable ni une motivation essentielle. Ce raisonnement est très utile pour examiner la fonction adaptative de la littérature (p. 138) : nous ne lisons pas des romans pour survivre, mais leur lecture est pourtant estimée favorable à la survie de l'espèce. David Shields illustre avec humour ce paradoxe dans son dernier livre intitulé How Literature Saved My Life. Aux yeux de l'auteur, il s'agit d'une antiphrase, car selon lui la littérature ne peut plus rien pour lui en tant qu'individu. Néanmoins, il affirme qu'elle peut au moins nous faire sentir en vie. Il cite à la fin de son livre Samuel Johnson: «Un livre devrait nous permettre soit d'échapper à l'existence soit de nous enseigner à la manière de l'endurer. » (p. 197, ma traduction) Il conçoit que l'art est peut-être la seule consolation face à la mort (p. 103), ce qui finalement reste assez darwinien.

\section{Historique de la question}

Je l'ai dit ailleurs (2012a, p. 343), c'est Taine (1828-1893) l'un des premiers à avoir proposé une forme de critique littéraire physiologique issue des découvertes de Darwin. Il s'agissait pour lui de remonter au mécanisme mental de l'écrivain en examinant la race (au sens d'«empreinte d'un esprit national »), le milieu (environnements physique et historique) et le moment particulier de l'œuvre. Fort de l'optimisme de Brunetière, qui déclare en conclusion de son Évolution de la 
poésie lyrique en France au dix-neuvième siècle : "Moi, je crois à l'évolution sur la parole des savants compétents, de Darwin ou d'Hæckel [...] parce que je vois que depuis une trentaine d'années, la théorie ou l'hypothèse a révolutionné, renouvelé, transformé l'histoire naturelle.» (t. 2, p. 290), et devant un silence embarrassé qui domine la quasi-totalité du $20^{\text {e }}$ siècle, faisons un grand saut pour en arriver au vif de notre sujet.

Car il a bien d'abord fallu poser la littérature comme le résultat ou la trace d'une adaptation chez homo sapiens, et ce concept est relativement récent. Les évolutionnistes de la dernière génération se sont penchés de près sur le statut adaptatif des narrations. La majorité d'entre eux s'accordent à dire que celles-ci ne sont pas le simple sous-produit d'un cerveau hyper-adapté, comme le prône en tête de file Stephen Pinker (voir sa critique dans Boyd, p. 81-83) Plutôt, en paraphrasant ce que dit clairement Michelle Scalise Sugiyama (p. 186), les récits auraient pour fonction de nous représenter des problèmes humains que nous rencontrons régulièrement, en regard des contraintes auxquelles sont confrontés les individus qui s'efforcent de les résoudre. Dans cette optique, il est remarquable que des romanciers énoncent à peu près la même chose sans pour autant se réclamer d'une théorie évolutionniste avouée. Exemple :

Le cinéma est une fenêtre sur l'âge adulte par laquelle on regarde cloué sur un fauteuil. On y apprend comment conduire une voiture en cas de poursuite, comment brandir une arme, comment embrasser sans maladresse une femme sublime; toutes choses que l'on ne fera pas mais qui comptent pour nous. C'est pour ça que l'on aime les fictions : elles proposent des solutions à des situations qui dans la vie sont inextricables; mais discerner les bonnes solutions des mauvaises permet de vivre. Le cinéma donne l'occasion de plusieurs vies. On voit, par 
la fenêtre hors d'atteinte, ce que l'on doit rejeter et ce qui doit nous être un modèle. Les fictions proposent comment faire, et les films que tout le monde a vus exposent les solutions les plus communes. Quand on s'assoit dans la salle on se tait, on voit ensemble ce qui a été, ce qui aurait pu être; ensemble. (Jenni, p. 480)

Ici, il est question de cinéma, mais on admettra aisément que ce propos s'applique tout autant à la littérature, avec laquelle le septième art entretient des relations très étroites. On peut avancer enfin que les progrès récents en neuroscience ne sont pas étrangers à ce vif intérêt porté par les évolutionnistes à tout ce qui relève des activités cognitives.

Posée donc cette hypothèse que la littérature dans sa forme la plus courante, les narrations, obéit à des principes adaptatifs, autrement dit représente un facteur crucial pour la survie de l'espèce humaine, quelle sorte de méthodologie devrons-nous façonner pour rendre compte du littéraire à travers la loupe de la psychologie évolutionniste?

Joseph Carroll (p. 77) est l'un des premiers Darwiniens à avoir véritablement posé les bases d'une méthodologie appliquée au texte littéraire. Il propose que n'importe quelle œuvre peut être vue sous le scialytique évolutionniste, selon une grille à cinq niveaux, dont les quatre premiers retiendront notre attention : une hiérarchie structurée des intentionnalités (motivation), le point de vue des consciences impliquées (auteur, personnages, lecteurs), le recours aux universaux humains et, enfin, les individualités au sein de catégories plus larges (la cinquième strate analytique concerne l'objet littéraire en tant que tel, jusqu'à ses procédés d'écriture). Dans l'article où est décrite cette grille analytique, Carroll se propose d'examiner Pride and Prejudice, le célèbre roman de Jane Austen 
(1813). Il insiste sur ce qu'il appelle les points de vue, qui subsument les trois niveaux cognitifs que sont l'auteur, ses personnages et les lecteurs. Il montre comment ces trois niveaux convergent en analysant le fameux incipit de ce roman comme suit :

It is a truth universally acknowledged that a single man in possession of a good fortune must be in want of a wife.

However little known the feelings or views of such a man may be on his first entering a neighbourhood; this truth is so well fixed in the minds of the surrounding families, that he is considered as the rightful property of some one or other of their daughters.

La première phrase énonce les règles du jeu universelles qui impliquent à la fois la recherche et l'obtention de la propriété et d'un partenaire (ici une femme) : c'est le point de vue déclaré de l'auteure. La seconde phrase, qui commence un nouveau paragraphe, amorce l'intrigue et concerne donc les personnages puisque c'est exactement ce qui va se passer : Bingley et Darcy, deux jeunes prétendants bien dotés, sont de passage dans le voisinage de la famille Bennet, qui compte cinq filles à marier, notamment les deux aînées, Jane et Elizabeth. Carroll explique enfin que la troisième phrase concerne les lecteurs, car puisque cette famille (la mère surtout) considère que ces deux jeunes hommes lui appartiennent déjà, elle est donc prédisposée à leur céder ses deux filles aînées. Elle montre ainsi un manque total de ce qu'on appelle la psychologie intuitive (theory of mind), mais que seuls les lecteurs peuvent deviner à ce point de l'histoire. Ce sont précisément ces préjugés qui sont dans le titre du roman et qui empêchent les unions harmonieuses de se former. Du point de vue narratif, le lecteur est donc pris au jeu dès le début, en particulier au niveau métacognitif. Non seulement il va lire une histoire fictive mais réaliste (niveau 
cognitif), mais il va devoir en conjecturer l'intrigue qui tourne uniquement autour des façons de percevoir les autres (niveau métacognitif). Le roman engendre des processus intellectuels qui mêlent des niveaux qu'on a naguère pris l'habitude de bien séparer. Nous les appellerons des foyers d'activité mentale ${ }^{4}$.

Cette vitrine des comportements typiquement humains en "période d'activité génitale» (jargon médical) va étaler devant nos yeux un très bon exemple de mésalliance, entre Lydia, la plus jeune des sœurs, écervelée et impulsive, et Wickham, un flambeur et joueur compulsif. Mais elle va aussi exhiber deux alliances plus réussies, quoique longues à mûrir, Jane avec Bingley et Elizabeth avec Darcy. À bon entendeur salut!

Ce qui est frappant dans le schéma de Carroll, c'est qu'il part justement du biologique. Ainsi l'hominidé que nous sommes ayant relativement peu évolué physiologiquement depuis quelque 10.000 ans, le premier niveau, celui de la hiérarchie structurée des intentionnalités, contient une séquence de systèmes comportementaux basiques: survie, technologie, accouplement, parentage, relations familiales, relations sociales, activités cognitives. Le deuxième niveau, celui des consciences impliquées dans l'histoire, pose comme prémisse fondamentale la recherche de ressources matérielles et de la reproduction réussie, non pas en tant qu'individu, mais pour l'espèce, car il y a bien évidemment toutes sortes de contre-exemples individuels à ces universaux. C'est à quoi répondent les deux niveaux suivants, à savoir la distinction

\footnotetext{
4 Ils recoupent plus ou moins le sens que l'on donnait au mot « esprit » à l'époque de Descartes (p. 8, n. 1) : «l'ensemble des facultés, à savoir la mémoire, la volonté, l'imagination, la sensibilité. »
} 
entre les caractères biologiquement communs à tous et les différenciations personnelles. Cependant, et pour reprendre l'exemple du roman d'Austen, on remarque que si le couple des parents Bennet n'est pas bien apparié, leurs deux filles aînées, Jane et Lizzy, vont, elles, contracter une alliance de qualité supérieure. À cet égard donc, force est de constater que la mésalliance des parents aura été bénéfique à long terme.

Ces schèmes sont si généraux qu'on pourrait y entrevoir une faiblesse rédhibitoire. Mais ce qui capte notre attention ici est précisément le fait d'envisager le texte littéraire au-delà du périmètre textuel à l'intérieur duquel la critique du dernier demi-siècle s'était ingéniée à l'enfermer. Le texte une fois éclaté, son auteur et ses lecteurs y retrouvent une place prépondérante autour des foyers d'activité mentale, ce qui ne contredit nullement les notions désormais acquises en narratologie : nul ne prétend que, dans un roman à la première personne, c'est l'auteur qui parle. Un jour que David Shields faisait partie du jury du National Book Awards pour les ouvrages de non-fiction, il a entendu un de ses collègues dénigrer un livre haut placé dans les scores, en arguant que son auteur n'arrêtait pas de s'immiscer dans l'histoire. Et Shields de rétorquer: «Mais qu'est-ce que cela veut-il dire? L'auteur qui s'immisce dans son histoire, c'est l'histoire, la meilleure histoire, la seule histoire. » (p. 30, ma traduction)

Si David Sloan Wilson (p. 30) n'hésite pas à parler de la nature quasi-génétique (genelike) des histoires, c'est bien parce que nos milliards de neurones ne cessent de nous reprogrammer afin de mieux nous adapter à toutes sortes de situations que nous rencontrons au fil des jours et que cette activité transite presque toujours par des narrations, effectives, 
auto-adressées ou mentales. Notre appartenance à « l'espèce symbolique », pour reprendre la notion phare de Terrence Deacon, a donc forcément consolidé, non seulement notre dépendance vitale au langage, mais notre propension à l'une des ses réalisations les plus abouties : la narration ${ }^{5}$.

\section{Pourquoi l'évolutionnisme en littérature : un exemple tiré d'un corpus récent}

Certes, les principes anthropologiques et biologiques qu'on peut appliquer à la littérature sont si généraux qu'on peut se demander à quoi ils vont bien nous servir. Nous avons affaire à une méthode empirique, qui ne saurait présumer des résultats qu'elle escompte a priori. Or quel avantage peut-on avancer en posant comme principe de départ que chaque histoire se fonde, à quelque niveau que ce soit, par la manifestation des caractéristiques universelles que sont la domination entre les mâles, la recherche du meilleur partenaire pour maximiser la reproduction et la sécurité, et éventuellement la soif de reconnaissance qui favorise en retour une meilleure adaptation inclusive ou indirecte ${ }^{6}$ ? Il se trouve pourtant que ces considérations surgissent aux endroits les plus inattendus. Ainsi au hasard des Gommes d'Alain Robbe-Grillet, livre phare

\footnotetext{
5 Selon Deacon, le cerveau humain se caractérise, comme celui des primates, par une préfrontalisation importante. Le langage s'est peu à peu développé de sorte que le cerveau s'est adapté à ces besoins neurocognitifs, en rapport aussi avec la motricité bipède et l'usage de nos bras et mains. Bref, ce n'est pas le cerveau qui a évolué pour nous permettre d'y caser le langage, mais le contraire : le cerveau a évolué pour s'adapter à notre besoin de langage, comme un instinct.

${ }^{6}$ Voir Workman et Reader, p. 42. Les Français conservent le terme fitness; je le traduis par adaptation.
} 
du Nouveau Roman, où l'on apprend que la femme de la victime, Daniel Dupont, a une quinzaine d'années de moins que lui. En se confiant à Wallas, le pseudo-inspecteur, elle lui avoue : «Pourquoi m'avait-il épousé?... Moi, j'étais très jeune et j'avais pour cet homme plus âgé [son professeur d'alors] une sorte d'admiration; tout le monde l'admirait autour de moi. » (p. 182) Point n'est donc besoin d'invoquer ici la rituelle armada freudienne, car la psychologie évolutionniste nous fournit des explications claires, sensées et parfaitement motivées dans une perspective darwinienne. Il ne s'agit pas de se contenter d'énoncer une banalité physiologique, mais au moins de constater que la psychologie évolutionniste offre une autre porte d'entrée à l'analyse textuelle: une jeune femme va naturellement se sentir attirée par un homme plus mûr, qui a peut-être même déjà des enfants, car il offre plus de gages de sécurité et d'expérience de vie qu'un jeune homme.

Pour terminer, je propose d'esquisser ce qui pourrait devenir une étude évolutionniste du dernier grand roman de Martin Winckler, Le Chœur des femmes (2009). Rappelons-en l'histoire dans ses grandes lignes. Jean Atwood termine son internat avec une spécialisation en chirurgie gynécologique. Première de sa classe durant tout son cursus universitaire, cette femme ambitieuse est vouée à une carrière brillante. Mais avant sa qualification, elle doit effectuer un dernier stage pratique dans un service hospitalier dirigé par un vieil ours barbu qui lui semble exagérément bienveillant. L'arrogance et la suffisance de la jeune interne n'échappent pas à "Barbe-Bleue», qui lui propose de valider son stage en une semaine au lieu de six mois si elle fait sans rechigner tout ce qu'il lui demandera de faire. Elle accepte à contrecœur, car elle n'aime pas se faire commander. On le devine, cette semaine va la transformer ou 
plutôt permettre de faire éclore le germe qu'elle avait déjà en elle, pour en faire potentiellement un médecin modèle.

Comme on est dans un centre de planification, il y est forcément question de contraception, d'IVG, de maternité, de naissance. Or le leitmotiv de la sexualité et de la reproduction est dans le roman en lutte constante avec la recherche du pouvoir absolu, exercé de manière abusive, pour ne pas dire totalitaire, de la part d'une poignée de personnes haut placées. On peut donc s'interroger sur les intentionnalités et les points de vue exprimés à différents niveaux. Ainsi l'avertissement liminaire pose sans ambigüité la posture de l'auteur : «Ce livre est un roman : les personnages, l'Unité 77, la ville de Tourmens, son CHU et les événements qui s'y déroulent sont imaginaires. Mais presque tout le reste est vrai. » Le roman est suivi de «notes bibliographiques» dans lesquelles Martin Winckler enfonce le clou: "Je ne saurais donc trop recommander aux personnes concernées ou intéressées par la question de l'intersexualité (et aux médecins qui ne veulent pas mourir idiots...) de consulter le site en anglais de l'ISNA (Intersex Society of North America) » (p. 673, mes italiques). Ce point de vue militant est en parfaite harmonie avec celui du Dr Franz Karma, chef de l'Unité 77, qui, tout en étant un personnage de fiction, semble dialoguer de bonne entente avec son géniteur. Par ailleurs, ce paratexte place directement les lecteurs de plain-pied avec les personnages de l'histoire; c'est plus que de la complicité, cela devient une participation active.

Cette postface révèle justement que l'écrivain a intégré une sélection parmi une multitude de courriels reçus via son site personnel, en les modifiant pour les besoins de la fiction. Or Martin Winckler précise qu'il « espère ne pas avoir dénaturé le 
sens de leurs paroles » (p. 674). Ces cris du cœur de patientes souvent inquiètes, mal informées, parfois maltraitées, résonnent fortement avec l'histoire dont c'est justement l'un des leitmotive. La soixantaine de ces lamentations confirme donc le «presque » de l'avertissement. Le lecteur est prévenu : l'histoire est imaginaire, mais pas son cadre ni sa toile de fond. Le fait que certains de ces courriels sont de pures inventions ne change rien à l'affaire : le discours reste le même sur l'échelle de la véracité.

Comment dès lors analyser ce roman en l'enfermant dans une diégèse dont l'auteur et le lecteur seraient de facto exclus, selon les principes narratologiques acquis depuis un bon demisiècle? Ce serait ignorer le pacte de lecture selon lequel il est explicitement dit que le point de vue du signataire de cette fiction entre directement en jeu dans les questions tournant autour des codes éthiques mis à mal au fil des pages. Les intentionnalités et points de vue de l'écrivain sont programmés dans le texte, où une poignée de personnages leur servent de vecteurs. Bien sûr, les personnages de roman ne sont pas des copies fidèles de personnes réelles, mais il est cependant tout à fait admissible de les considérer jusqu'à un certain degré comme des porte-parole ou des hérauts d'une certaine idéologie7. Dans Le Chœur des femmes, on assiste à la lutte constante et âpre entre la recherche du pouvoir et de la renommée (les "chefs» en gynécologie) d'un côté, qui ne s'embarrasse guère de bafouer l'éthique des soins, et de l'autre,

\footnotetext{
7 «Et quand certains critiques font la moue en disant que mes personnages sont manichéens, ça me fait rire. Un personnage, ça ne peut jamais être aussi fin et complexe qu'une personne réelle, et je n'ai pas la vanité de croire que les miens le sont! Mais je ne crois pas non plus que tous les romans aient pour but de faire de la psychologie fine. » (Winckler, 2010, p. 99)
} 
la quête sans cesse réapprise de l'exercice médical dans le pur respect de l'individu, patient ou collègue, ici représenté par le docteur Franz Karma, hommage lointain (et avoué) au Barberousse du film du même nom du réalisateur japonais Kurosawa (1965).

Comme pour le roman de Jane Austen, l'incipit est très révélateur. La première phrase de l'« Ouverture » est : «Qu'estce qu'on m'avait raconté, déjà? » (p. 17). Le personnage central, Jean Atwood, est sur le point d'entrer au service de BarbeBleue; elle tente de se remémorer les «histoires » qu'on lui a racontées. Suivent une litanie de rumeurs plus ou moins fausses à son égard, évidemment exagérées par l'arrogance de la narratrice : orgueil et préjugés! Tout comme Elizabeth dans le roman de Jane Austen, Jean va évoluer pendant l'histoire pour ajuster sa psychologie intuitive, déficiente non seulement vis-àvis du personnel du service dans lequel elle va passer une semaine - elle traite son supérieur avec le mépris d'une jeune arriviste, et ses inférieurs comme des objets -, mais aussi à l'égard de son amant Joël, dont elle a pris la prévenance pour de la manipulation.

Le point de vue, que nous pourrions ici assimiler à un foyer d'intentionnalité, pourrait aussi s'appliquer aux personnages « vertueux » qui, eux aussi, doivent s'adapter à un environnement sans cesse changeant. Ainsi de Franz Karma, le gourou de l'Unité 77 , sur qui pèse un lourd fardeau relié au passé de Jean. Dans Le Chœur des femmes, tous les personnages tendent vers une harmonisation liée à l'évolution de leur psychologie intuitive respective.

La psychologie évolutionniste ne prétend pas répondre à tout, ni résoudre de manière formelle tous les enjeux 
romanesques de la fiction. Elle offre toutefois une nouvelle porte d'entrée à son analyse et remet l'humain là ou il n'a jamais cessé d'exister et de prospérer : la littérature. Le dernier mot revient à Jane Austen, dont le personnage de Jean Atwood aurait bien fait de suivre à la lettre la leçon suivante, énoncée en termes darwiniens, par anticipation évidemment :

"A woman must have a thorough knowledge of music, singing, drawing, dancing, and the modern languages, to deserve the word ["accomplished"]; and besides all this, she must possess a certain something in her air and manner of walking, the tone of her voice, her address and expressions, or the word will be but half deserved. »

« All this she must possess », added Darcy, « and to all this she must yet add something more substantial, in the improvement of her mind by extensive reading. » (p. 33)

\section{Bibliographie}

Appleman, Philip (ed.). (1970), Darwin: A Norton Critical Edition, New York, Norton \& Co.

Austen, Jane. (1946), Pride and Prejudice, Londres, the Zodiac Press.

BARLOW, Nora, Ed. (2005), The Autobiography of Charles Darwin, New York, Londres, W.W. Norton.

BoyD, Brian. (2009), On The Origin of Stories: Evolution, Cognition and Fiction, Cambridge MA, Londres, The Belknap Press of Harvard University Press. 
BRUNETIÈRE, Ferdinand. (1895), L'Évolution de la poésie lyrique en France au dix-neuvième siècle, Paris, Librairie Hachette, 2 tomes).

CARroll, Joseph. (2005), « Human Nature and Literary Meaning: A Theoretical Model Illustrated with a Critique of Pride and Prejudice », The Literary Animal, p. 76-106.

DEACon, Terrence. (1997), The Symbolic Species, New York, Norton \& Co.

DeSCARTES, René. (2007), Discours de la méthode, Paris, Hatier Poche.

GHISELIN, Michael T. (2003) The Triumph of the Darwinian Method, Mineola, New York, Dover Publications.

GoTTSCHALL, Jonathan et David Sloan WILSON (ed.). (2005), The

Literary Animal: Evolution and the Nature of Narrative, Evanston (Illinois), Northwestern University Press.

JENNI, Alexis. (2011), L'Art français de la guerre, Paris, Gallimard.

LAPPRAND, Marc. (2012a), « Pour une approche bioculturelle des récits », @nalyses, vol. 7, no 3, p. 342-364,

$<$ http://www.revue-analyses.org/>.

- . (2012b), Trois pour un : une lecture évolutionniste de l'œuvre de Martin Winckler, Québec, Presses de l'Université du Québec.

RIDLEY, Mark (ed.). (1987), The Essential Darwin, Londres, Sydney, Unwin Paperbacks.

RobBe-Grillet, Alain. (1953), Les Gommes, Paris, Minuit.

SCAlise SugiYAmA, Michelle. (2005), «Reverse-Engineering Narrative: Evidence of Special Design », The Literary Animal, p. 177-196.

SHIELDS, David. (2013), How Literature Saved My Life, New York, Alfred A. Knopf. 
SYMONDS, John Addington. (1970), «On the Application of Evolutionary Principles to Art and Literature », dans Philip Appleman (ed.). Darwin: A Norton Critical Edition, New York, Norton \& Co, p. 605-611.

WILSON, David Sloan. (2005), «Evolutionary Social Constructivism », The Literary Animal, p. 20-37.

WiNCKLER, Martin. (2009), Le Chœur des femmes, P.O.L, coll. «Folio ».

—. (2010), « Le Médecin-écrivain, l'éthique et l'imaginaire, » Les ateliers de l'éthique : revue du CREUM, vol. 5, no 1, p. 83-100. Workman, Lance et Will READER. (2007), Psychologie évolutionniste: Une introduction, Bruxelles, De Boeck (traduction de Evolutionary Psychology: An Introduction, Cambridge, Cambridge University Press, 2004).

\section{Résumé}

Joseph Carroll fait partie des pionniers du darwinisme littéraire. Il propose une méthode analytique fondée sur les pôles narratifs qui englobent l'auteur, les lecteurs et les personnages (sans pour autant les mettre au même niveau). Mais à constamment dévoiler, chemin faisant, les universaux biologiques de l'espèce humaine, on peut se demander in fine si cette méthode ne s'applique pas à tout sans produire nécessairement de nouvelles interprétations révolutionnaires du texte littéraire. Malgré tout, et c'est ce à quoi cet article tente de s'attacher, nous sommes en présence d'un nouveau champ de la connaissance qui, à la faveur des avancées récentes dans les sciences neurocognitives, s'implante comme une épistémologie qui renouvelle de manière salutaire la critique littéraire. Il sera proposé en fin d'article une esquisse d'analyse évolutionniste d'un roman contemporain, Le Chœur des femmes de Martin Winckler. 


\begin{abstract}
Joseph Carroll is one of the pioneering researchers on literary Darwinism. He proposes an analytical method based on narrative poles which encompass the author, the readers and the characters (without putting them quite at the same level). But, while constantly and step by step unveiling human universals, one may wonder whether in the end this ideology cannot be applied to anything without necessarily producing new or revolutionary textual interpretations of literature. Still, and it is one of the aims of this article, we are faced with a new field of knowledge which, in the wake of recent forays in neurocognitive sciences, is establishing itself as another epistemology aiming at renewing literary criticism in a healthy manner. The conclusion of this paper will propose the outline of an evolutionary analysis of a contemporary novel, Le Chœur des femmes, by Martin Winckler.
\end{abstract}

